\title{
Porous-based rheological model for tissue fluidisation
}

\author{
N. Asadipour ${ }^{\mathrm{a}}$, X.Trepat ${ }^{\mathrm{b}, \mathrm{c}}$, J.J. Muñoz ${ }^{\mathrm{a}, \mathrm{d}}$ \\ ${ }^{a}$ Laboratori de Càlcul Numèric (LaCàN), Dept. Mathematics, Esc. Univ. d'Eng. Tèc. \\ Industrials (EUETIB), Universitat Politècnica de Catalunya-Barcelona Tech (UPC), \\ 08036, Barcelona, Spain \\ ${ }^{b}$ Institut Català de Recerca i Estudis Avançats (ICREA), Barcelona 08028, Spain and \\ Institute for Bioengineering of Catalonia, Barcelona 08028, Spain. \\ ${ }^{c}$ Centro de Investigación Biomédica en Red en Bioingeniería, Biomateriales y \\ Nanomedicina. Facultat de Medicina, Universitat de Barcelona, 08036 Barcelona, Spain. \\ ${ }^{d}$ Barcelona Grad. School of Mathematics (BGSMath)
}

\begin{abstract}
It has been experimentally observed that cells exhibit a fluidisation process when subjected to a transient stretch, with an eventual recovery of the mechanical properties upon removal of the applied deformation. This fluidisation process is characterised by a decrease of the stored modulus and an increase of the phase angle. We propose a rheological model which is able to reproduce this combined mechanical response. The model is described in the context of continua and adapted to a cell-centred particle system that simulates cell-cell interactions. Mechanical equilibrium is coupled with two evolution laws: (i) one for the reference configuration, and (ii) another for the porosity or polymer density. The first law depends on the actual strain of the tissue, while the second assumes different remodelling rates during porosity increase and decrease. The theory is implemented on a particle based model and tested on a stretching experiment. The numerical results agree with the experimental measurements for different stretching magnitudes.
\end{abstract}

Keywords: fluidisation, viscoelasticity, softening, cell remodelling, cell rheology

\section{Introduction}

Cells are complex structures consisting of a wide number of binding proteins and other solid and fluid constituents. Mechanically, they are stabilized by the cytoskeleton, a highly active contractile polymeric network which is 
able to (de)polymerise and reinforce [9]. This cytoskeletal activity renders cells complex rheological responses, exhibiting stiffening [7, 9, 33] and softening [37]. Due to a reduction of the phase angle upon stretching, it is also considered that cells undergo fluidisation, either individually [13] or when forming a soft tissue [34]. We here propose a rheological model for such softening and fluidisation process, which is also able to simulate the eventual recovery upon removal of the applied stretch.

Material fluidisation has been associated to apoptotic events and cell division [27]. We instead restrict our attention to tissues with a constant number of cells and no connectivity changes, as it has been observed in in vitro experiments $[13,34]$. Other experiments have revealed the reversibility of the softening and stiffening during a stretch cycle, and explained this behaviour through the presence of motor proteins [3] or the geometrical orientation of the fibers [12]. We focus instead on the delayed recovery of the material properties after the stretch cycle. This response has been already explained in the context of soft glassy framework using a Glassy Wormlike Chain model (GWLC) [9], which has been extended for modelling material softening [37]. These authors propose a model based on a non-linear form of the GWLC model based on crosslinked biopolymer networks, where bond fraction kinetics is accounted for.

Since we aim to implement the softening behaviour on arbitrary multicellular systems, and include the resulting rheological law on general cell topologies, we present here an alternative model based on an extension of a Maxwell-like law that uses an adaptive resting length [22]. We complement the model with a suitable evolution of an additional variable that represents the polymer density. This porosity-like variable mediates the mechanical response of the cell and controls the effective stress that cells can exert, in a similar manner to the damage variable in continuum mechanics. The modulation of softening with damage variables has already been formulated for instance in $[24,25]$. In our model, we relate cell stiffness with the porosity and damage variables in a similar manner as it has been experimentally measured [19], but in contrast to damage models, the damage-like is allowed to decrease due to recovery of initial porosity.

The relevance of porosity in living tissues has already been considered in early models of cardiac muscle [39]. More recently, it has been applied for simulating polymeric materials [35], or when assuming a biphasic material consisting of a porous cytoskeletal network [19]. Other continuous models for rubbers took into account the molecular chain density (or fraction of 
soften versus stiff material) [26], or the fibre and matrix contributions through internal variables $[2,16,30]$. These models are based on damage variables or phase transitions, and only consider irreversible softening. In our case instead the material stiffness is eventually recovered, as our formulation also captures.

\section{Continuum model}

The explicit modelling of the filaments in the cytoskeleton would render the resulting model computationally expensive if not prohibitive for analysing multicellular systems. For this reason, we idealise first in this section the observed domain $\Omega \in \mathrm{R}^{3}$ as a continuum, whose shape is the result of an active and an elastic deformation. Computationally, we express this two contributions by decomposing the deformation gradient [10, 21, 28, 29], where each material point $\boldsymbol{X}_{0} \in \Omega_{0}$ moves to a new position $\boldsymbol{x} \in \Omega(t) \in \mathrm{R}^{3}$. The total deformation gradient $\boldsymbol{F}=\frac{\partial \boldsymbol{x}}{\partial \boldsymbol{X}_{0}}$ is decomposed in an active and an elastic deformation, respectively denoted by $\boldsymbol{F}^{a}=\frac{\partial \boldsymbol{x}^{a}}{\partial \boldsymbol{X}_{0}}$ and $\boldsymbol{F}^{e}=\frac{\partial \boldsymbol{x}}{\partial \boldsymbol{x}^{a}}$, and such that $\boldsymbol{F}=\boldsymbol{F}^{e} \boldsymbol{F}^{a}$.

The vector $\boldsymbol{x}^{a} \in \Omega^{a}$ denotes the position of the material points prior to any elastic deformation, with $\Omega^{a}$ a relaxed configuration, not necessarily observed or compatible with the material impenetrability assumption. This incompatibility assumes that the vector field $\boldsymbol{x}^{a}$ is piecewise continuous. At the regions where it is discontinuous, the definitions $\boldsymbol{F}^{a}=\frac{\partial \boldsymbol{x}^{a}}{\partial \boldsymbol{X}_{0}}$ and $\boldsymbol{F}^{e}=$ $\frac{\partial \boldsymbol{x}}{\partial \boldsymbol{x}^{a}}$ are not valid, and $\boldsymbol{F}^{a}$ and $\boldsymbol{F}^{e}$ must be assumed as non-smooth twopoint tensors. We use the configuration $\Omega^{a}$ to represent the remodelling and internal reorganisation of the biologically active material.

The description of the cell activity is represented in this paper as a function of a parameter $P$ that represents the polymer porosity or void content. The resulting model is based on three basic ingredients:

- Mechanical equilibrium of the observed domain $\Omega$.

- Evolution law for the reference configuration $\Omega^{a}$, as a function of the elastic deformation $\boldsymbol{F}^{e}$ and polymer porosity $P$.

- Evolution law for the polymer porosity $P$.

The second ingredient reflects the ability of cells to adapt to the imposed deformations, while the third ingredient aims to mimic the evolution of the 
polymeric network without explicitly representing the elements of the cytoskeleton. We will next detail each one of the three ingredients given above.

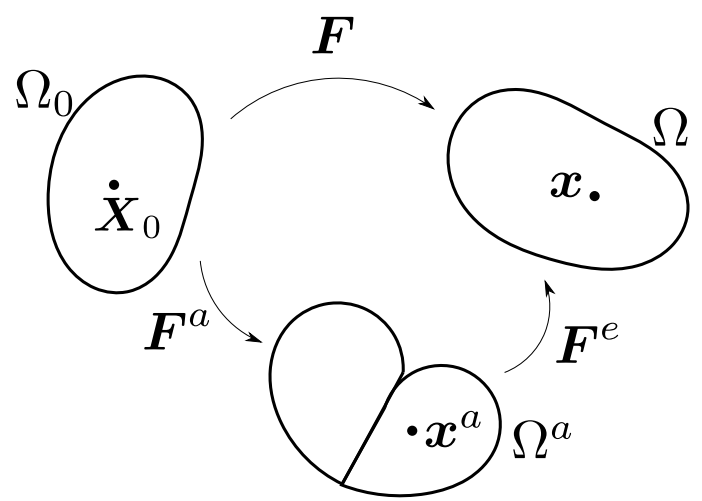

Figure 1: Schematic of the multiplicative decomposition of the total deformation gradient, $\boldsymbol{F}$, in a growth component, $\boldsymbol{F}^{a}$, and elastic component, $\boldsymbol{F}^{e}$, i.e. $\boldsymbol{F}=\boldsymbol{F}^{e} \boldsymbol{F}^{a}$.

\subsection{Mechanical equilibrium}

Similarly to the damage models, we consider a free energy function which is mediated through a damage-like parameter $D$,

$$
U(\boldsymbol{C}, D)=(1-D) U^{e}(\boldsymbol{C}),
$$

where $U^{e}(\boldsymbol{C})$ is the strain energy function. The latter is assumed to depend on the right Cauchy Green tensor $\boldsymbol{C}=\boldsymbol{F}^{\boldsymbol{e} T} \boldsymbol{F}^{e}$, with $\boldsymbol{F}^{e}=\frac{\partial \boldsymbol{x}}{\partial \boldsymbol{x}^{a}}$ the deformation gradient tensor. Parameter $D$ is assumed to depend on a porosity variable $P$ as,

$$
D=c_{1} \frac{P-P_{0}}{P_{0}} .
$$

with $c_{1}$ a positive material constant. Variable $P$ represents the porosity or void content of the cross-linked network of the cytoskeleton, and $P_{0}$ is a reference value. $P$ may be interpreted as the inverse of the polymer density or entanglement of the cytoskeleton. We will assume that both $P$ and $P_{0}$ are non-negative, i.e. $P, P_{0} \geq 0$, and also that $0 \leq P \leq P_{0}\left(1+c_{1}\right) / c_{1}$. Therefore, and unlike damage models, $D$ may have negative values, and $-c_{1} \leq D \leq$ 1. The case $D \leq 0$ represents stiffening due to increased entanglement or increase of polymer density. 
The equilibrium equations of the continuum $\Omega$ are given by Cauchy equation [11]:

$$
\nabla \cdot\left(\left(J^{e}\right)^{-1} \boldsymbol{F}^{e} \boldsymbol{S} \boldsymbol{F}^{e T}\right)=\mathbf{0}, \forall x \in \Omega,
$$

where $\boldsymbol{S}$ is the second Piola-Kirchhoff stress tensor, and $J^{e}=\operatorname{det}\left(\boldsymbol{F}^{e}\right)$. The relation between $\boldsymbol{S}$ and $U(\boldsymbol{C}, D)$ follows from the Clausius-Duhem inequality for an isothermal material [18],

$$
\frac{1}{2} \boldsymbol{S}: \dot{\boldsymbol{C}}-\dot{U} \geq 0
$$

The rate of free energy can be attributed the variations of $P$ and $\boldsymbol{C}$, but for living materials, also to other energy sources such as ATP hydrolysis or binding of adhesive complexes, which will be here represented by the quantity $N_{c}$. In this case, the inequality above implies that,

$$
\begin{array}{r}
\boldsymbol{S}=2 \frac{\partial U}{\partial \boldsymbol{C}}=2(1-D) \frac{\partial U^{e}}{\partial \boldsymbol{C}} \\
\frac{\partial U}{\partial N_{c}} \dot{N}_{c}+\frac{\partial U}{\partial P} \dot{P} \leq 0
\end{array}
$$

Note that from the expression of $U$ in (1)-(2), the previous inequality reads,

$$
c_{1} \frac{\dot{P}}{P_{0}} U^{e}(\boldsymbol{C}) \geq \frac{\partial U}{\partial N_{c}} \dot{N}_{c} .
$$

Since $c_{1}, P_{0}>0$ and $U^{e}(\boldsymbol{C}) \geq 0$, we have that $\dot{P} \geq \alpha \frac{\partial U}{\partial N_{c}} \dot{N}_{c}$, with $\alpha>0$, when the tissue is deformed $\left(U^{e}>0\right)$. For a dead material $\left(\dot{N}_{c}=0\right)$, the porosity $P$ and damage variable $D$ may only increase, but for a living material, this is not necessarily so, and $\dot{P}$ can change sign, depending on the rate of adhesive complexes and cell activity. A more detailed description of the mechanical balance of the cytoskeleton and its thermodynamical equilibrium can be found in [17]. We will here propose an evolution law for $P$ in Section 2.3 that actually allows for positive and negative rates of porosity.

The variations of the stress response have effects on the strain energy, which is modulated by this porous-like variable. We assume that the available energy of the active material ("metabolic" energy) is unbounded, so that cell activity is energetically unconstrained. This hypothesis is based on estimations made on a set of tissues and strain rates [5], which include our range $\dot{\varepsilon} \approx 0.025-0.05 s^{-1}$, common in the lung tissues studied here. 


\subsection{Evolution of active configuration}

The actin cytoskeleton is a composite intracellular biopolymer network made of actin filaments that can actively vary their length and entanglement. Since these changes may strongly affect cellular rheology, we include here an evolution law for the active deformation of the polymers, $\boldsymbol{E}^{a}=\frac{1}{2}\left(\boldsymbol{F}^{a T} \boldsymbol{F}^{a}-\boldsymbol{I}\right)$. This deformation may be due to multiple phenomena such as transient domain unfolding [6], remodelling of cytoskeletal filaments [4], un/rebinding of sticky contacts [32], activity of crosslinking molecules $[14,38]$, forming of actin-myosin cross bridges [12], or (de)polymerisation $[1,15]$.

We will not detail the specific causes behind the changes in the polymer lengths, which we mechanically identify with a time-varying active deformation $\boldsymbol{E}^{a}$ that satisfies the following evolution law:

$$
\dot{\boldsymbol{E}}^{a}=\gamma: \boldsymbol{E}^{e},
$$

with $\boldsymbol{E}^{e}=\frac{1}{2}\left(\boldsymbol{F}^{e T} \boldsymbol{F}^{e}-\mathbf{I}\right)$ the Green-Lagrange elastic strain tensor. Tensor $\gamma$ represents the resistance of the network to adapt its relaxed configuration to the current elastic strain and will be called the remodelling rate.

The physical picture behind the evolution law in (4) is a network of cross-linked actin filaments (Figure 2a) that when subjected to a macroscopic strain, it stretches mainly as a result of two combined phenomena: (i) a reversible (elastic) deformation and a (ii) non-reversible remodelling and lengthening, due to the (de)polymerisation process of the filaments.

(a) Cross-linked actin filament network

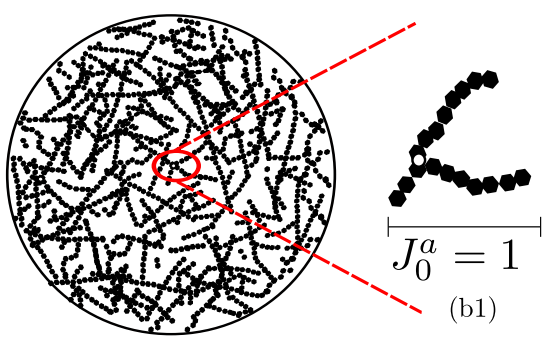

(b) Reduced system with two filaments and a cross-link

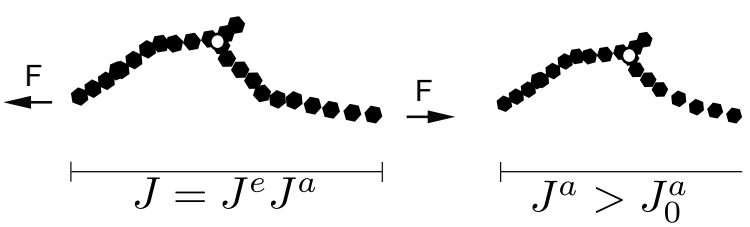

(b2) (b3)

Figure 2: (a) Schematic of network of actin filaments connected by flexible cross-links. (b) Schematic of strain induced changes in the active deformation $J^{a}=\operatorname{det}\left(\boldsymbol{F}^{a}\right)$ of a reduced system with two filaments and a cross-link (white circle). (b1) Initial configuration with active deformation $J_{0}^{a}=1$. (b2) Configuration under an applied load. (b3) New unstrained configuration with modified active deformation $J^{a}>J_{0}^{a}$. 
Similarly to the elasticity tensor, we propose an isotropic form of the remodelling rate tensor as $\gamma_{i j k l}=\gamma_{1} \delta_{i j} \delta_{k l}+\gamma_{2}\left(\delta_{i k} \delta_{j l}+\delta_{i l} \delta_{j k}\right)$, with $\gamma_{1}$ and $\gamma_{2}$ scalar parameters. In this case, due to the symmetry of $\boldsymbol{E}^{e}$, the evolution law in (4) reduces to,

$$
\dot{\boldsymbol{E}}^{a}=\gamma_{1} \operatorname{tr}\left(\boldsymbol{E}^{e}\right)+2 \gamma_{2} \boldsymbol{E}^{e} .
$$

When $\gamma_{1}=0$, the form above is tantamount to applying an active deformation which is component-wise proportional to the elastic deformation, or when $\gamma_{2}=0$, which is proportional to the linearised volumetric elastic deformations. Alternatively, if some conditions on the total deformations $\boldsymbol{F}$ are considered, the amount of active deformation may be accordingly constrained, restricting the form of tensor $\boldsymbol{\gamma}$. For instance, if the constraint $\operatorname{det}(\boldsymbol{F})=1$ applies, we must have that $\dot{J}=\dot{J}^{a} J^{e}+J^{a} \dot{J}^{e}=0$. In this case, since,

$$
\dot{J}^{a}=J^{a} \operatorname{tr}\left(\frac{\partial \boldsymbol{v}^{a}}{\partial \boldsymbol{x}^{a}}\right)=J^{a} \nabla_{x_{a}} \cdot \boldsymbol{v}^{a},
$$

with $\boldsymbol{v}^{a}=\left.\frac{d \boldsymbol{x}^{a}}{d t}\right|_{\boldsymbol{X}_{0}=\text { const }}$, the following relation must hold:

$$
\nabla_{x^{a}} \cdot \boldsymbol{v}^{a}=-\dot{J} e / J^{e} .
$$

This constraint may be combined with the relationship

$$
\operatorname{tr}\left(\boldsymbol{F}^{a-T} \dot{\boldsymbol{E}}^{a} \boldsymbol{F}^{a-1}\right)=\nabla_{x^{a}} \cdot \boldsymbol{v}^{a},
$$

giving rise to the following kinematic condition:

$$
-\dot{J}^{e} / J^{e}=\operatorname{tr}\left(\boldsymbol{F}^{a-T} \dot{\boldsymbol{E}}^{a} \boldsymbol{F}^{a-1}\right) .
$$

This relation is not automatically satisfied if the simple linear evolution form in (4) is employed. However, and in view of the condition above, a more sophisticated evolution law such as $\dot{\boldsymbol{E}}^{a}=-\frac{j^{e}}{3 J^{e}} \boldsymbol{F}^{a T} \boldsymbol{F}^{a}$ would guarantee the isochoric constraint $\dot{J}=0$. For simplicity, and when considering one-dimensional implementations, we will restrict our presentation to linear evolution laws with the form in (4).

In addition to the evolution law in (4), filament density has been also identified as an important parameter in mechanical weakening [31]. This dependence is mathematically described with the following simple relationship,

$$
\gamma=P \gamma_{0},
$$


where $\gamma_{0}$ is considered a constant remodelling rate. Figure 3 illustrates this dependence. As the porosity $P$ increases (or cell density decreases), the cytoskeleton adapts more easily its resting configuration, and conversely, as the porosity decreases, more filaments resist to adapt the resting configuration.

The relations in (4)-(5) are simple linear laws, but without further experimental evidence, it seems as yet unnecessary to test more complicated relations. The main implications of the proposed laws are,

(i) No internal remodelling occurs if the filament is not subjected to stretch,

(ii) The filament tends to reduce the amount of elastic strain energy.

(iii) The remodelling rate increases as the polymer density decreases.

\subsection{Cell porosity evolution}

Since we do not aim to explicitly model the polymer network, but to characterise this network through its porosity and resting length, we propose here the following relation for the evolution of $P$ :

$$
\dot{P}=c_{2}^{ \pm}\left(P_{0}-\frac{P}{\left(J^{a}\right)^{c_{3}}}\right),
$$

with $J^{a}=\operatorname{det}\left(\boldsymbol{F}^{a}\right)$, and $P_{0}$ a reference homeostatic value of the porosity, at which no further changes on $P$ are induced. Equation (6) represents a self-regulation of the void content, where the material constants $c_{2}^{ \pm}$and $c_{3}$ determine how strongly this self-regulation takes place, and how strongly $\dot{P}$ depends on the active deformation $J^{a}$. We further hypothesise that $c_{2}^{ \pm}$may take different values dependent on whether $P$ is increasing $\left(c_{2}^{ \pm}=c_{2}^{+}\right)$or $P$ is decreasing $\left(c_{2}^{ \pm}=c_{2}^{-}\right)$, with $c_{2}^{+}>c_{2}^{-}$. The physical hypotheses in (6) are the following:

- Networks with larger resting configurations induce an increase of the porosity (filaments tend to stay farther from each other) while smaller resting domains render the polymeric network denser. This is illustrated in Figure 3. The dependence of $\dot{P}$ on $J^{a}$ is plotted in Figure $4 \mathrm{a}$.

- When the porosity is below the homeostatic state $P_{0}\left(J^{a}\right)^{c_{3}}$, the porosity will tend to increase, and vice-versa. Figure $4 \mathrm{~b}$ illustrates this dependence. 
- Unsymmetrical polymer remodelling: porosity recovers its reference value $P_{0}\left(J^{a}\right)^{c_{3}}$ much easily when $P$ is increasing than when it is decreasing.

We note that a somewhat similar relation as in the first hypothesis has been experimentally confirmed [19], where a longer entanglement length has been associated with an increase of the porosity and a reduction of tissue stiffness.

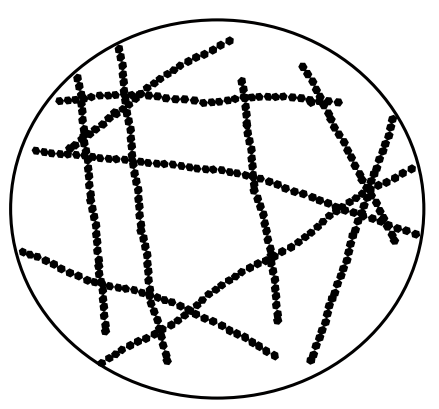

(a)

$J^{a}>1$

$P>P_{0}$

$D>0$

$\gamma>P_{0} \gamma_{0}$

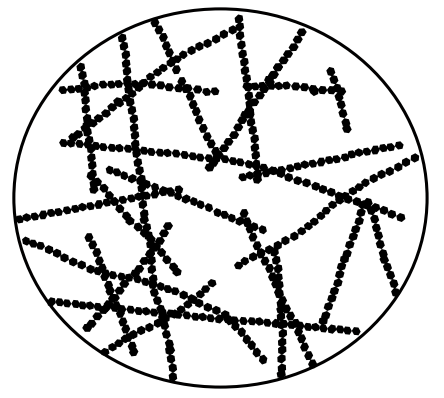

(b)

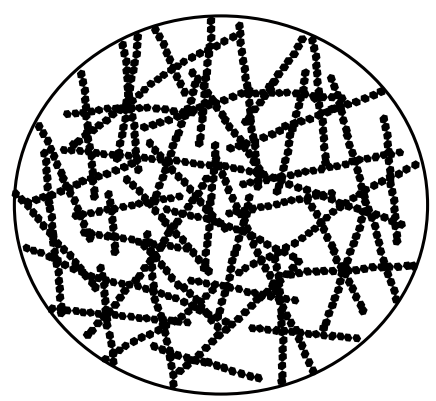

(c)

$J^{a}=1$

$J^{a}<1$

$P=P_{0}$

$P<P_{0}$

$D=0$

$D<0$

$\gamma=P_{0} \gamma_{0}$

Figure 3: Physical illustration of equations (2), (5) and (6):(a) When the resting volume increases $\left(J^{a}>1\right)$, the material softens and the porosity increases $(\dot{P}>0)$, and eventually diminishes when $P_{0}-P /\left(J^{a}\right)^{c_{3}}<0$. (b) Initial configuration of a system of actin filaments with resting volume equal to $J_{0}^{a}=J_{0}=1=\operatorname{det}\left(\boldsymbol{F}^{e}\right)$. (c) By decreasing the resting volume $\left(J^{a}<1\right)$, the material stiffens and the porosity decreases $(\dot{P}<0)$ due to the reduction of $J^{a}$, and eventually increases when $P_{0}-P /\left(J^{a}\right)^{c_{3}}>0$.

\subsection{Summary of the model}

The continuum model described so far can be summarised in the three equations (3), (4) and (6), which may be gathered in a system of PDEs:

$$
\left\{\begin{array}{l}
\nabla \cdot\left(\left(J^{e}\right)^{-1} \boldsymbol{F}^{e} \boldsymbol{S} \boldsymbol{F}^{e T}\right)=\mathbf{0}, \forall \boldsymbol{x} \in \Omega \\
\dot{\boldsymbol{E}}^{a}=P \boldsymbol{\gamma}_{0}: \boldsymbol{E}^{e} \\
\dot{P}=c_{2}^{ \pm}\left(P_{0}-\frac{P}{\left(J^{a}\right)^{c 3}}\right)
\end{array}\right.
$$



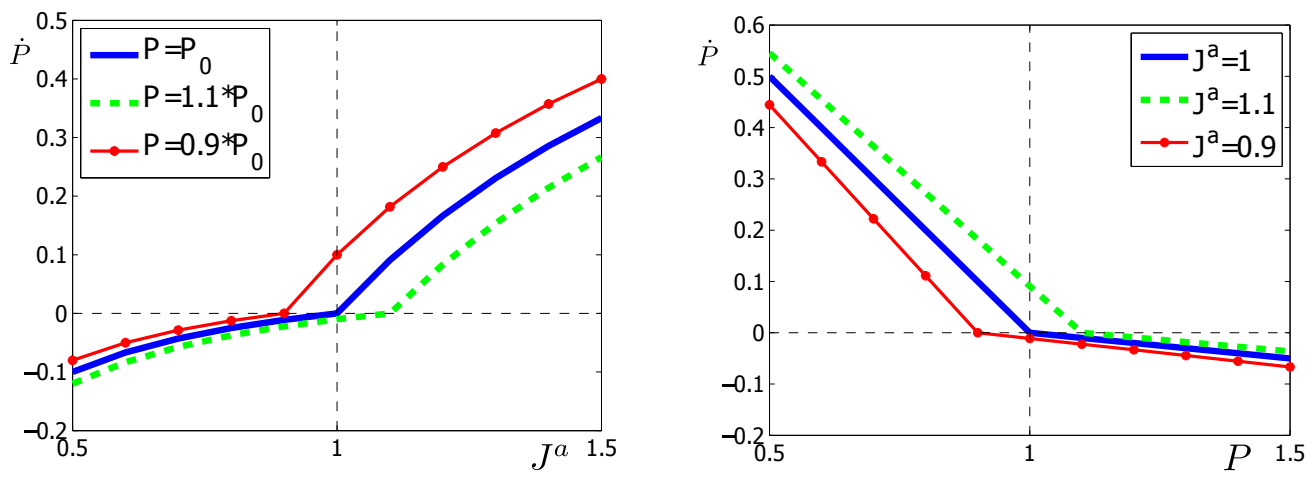

Figure 4: Evolution of $P$ according to equation (6) with $c_{3}=1$, and $c_{2}^{+} / c_{2}^{-}=10$. (a) Rate of cell porosity, $\dot{P}$, against the resting $J^{a}$, for $P_{0}=1$, and different values of $P$. $\dot{P}$ decreases when the resting volume decreases $\left(J^{a}<1\right)$. (b) Rate of cell porosity, $\dot{P}$, as a function of $P$ for $P_{0}=1$, and different values of $J^{a}$.

where

$$
\boldsymbol{S}=2 \frac{\partial U}{\partial \boldsymbol{C}} \quad, \quad U(\boldsymbol{F})=(1-D) U^{e}(\boldsymbol{F}) \quad, \quad D=c_{1} \frac{P-P_{0}}{P_{0}}
$$

According to the expression of the damage factor $1-D=1+c_{1}-c_{1} \frac{P}{P_{0}}$ and the evolution of $\dot{\boldsymbol{E}}^{a}$, if the porosity increases while keeping the elastic deformation $\boldsymbol{E}^{e}$ constant, the stress $\boldsymbol{S}$ will decrease but the active strains $\boldsymbol{E}^{a}$ will increase. Indeed, and as depicted in Figure 3a, if the density of filaments is lower, then the total stress carried by all the filament should decrease, and the cell also adapts more easily to the current elastic deformation. Conversely, a reduction in cell porosity causes an increase in the stresses (for a given elastic deformation) and a decrease of the active strains due to a reduction of polymer remodelling (see Figure 3c).

We note that we are not explicitly modelling fluid flow in the porous biological material, and so this is not a so-called poroelastic model. However, porosity is considered as an internal variable which modulates the effective stress, but without considering the transfer of stresses between fluid and solid phases.

The formulation in (7) formally satisfies the physical hypotheses, but its not straightforward to implement in the continuum context. The values in the fourth-order tensor $\gamma_{i j k l}$, which represent the rate of a given active strain in the components $i j$ when subjected to an elastic strain in the $k l$ component cannot be easily measured experimentally. For this reason we 
present in the next section a one dimensional version, which would contain far less parameters and that can be implemented in a truss model of the tissue.

\section{Computational implementation on cell-centred model}

The equations of the continuum model described in the previous section are here adapted to a particle based model in order to simplify the evolution law for $\dot{\boldsymbol{E}}^{a}$. Figure 5 shows a schematic of the cell-centred model that will be employed to simulate the fluidisation phenomena. Each cell-centre (nucleus) is represented as a particle $\boldsymbol{x}^{i}, i=1, \ldots, N$, and the cell-cell interactions are implemented on $N_{e}$ bar elements that join the particles using a Delaunay triangulation of the initial particle positions. The Voronoi tessellation of the Delaunay triangulation (see Figure 5b) may be used to represent the cell-cell boundaries. In this work though, we will not make use of these boundaries in our equations.

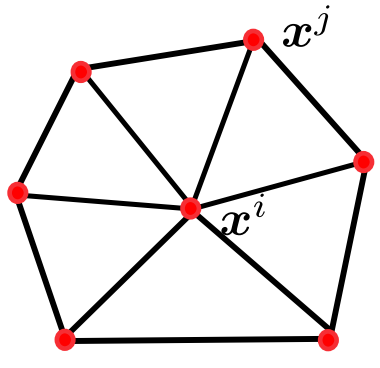

(a)

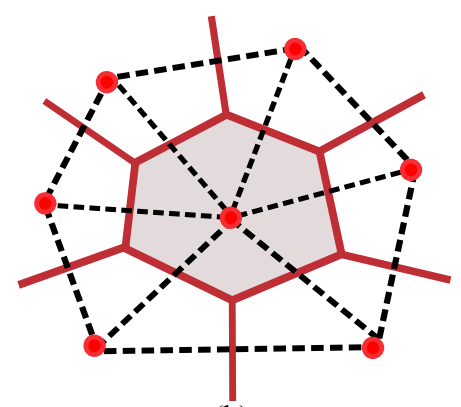

(b)

Figure 5: A schematic view of the truss model: (a) Delaunay triangulation of the cellcentres (black lines). (b) Voronoi tessellation representing the cell boundaries (red lines).

We remark that in the discrete cell-centred representation that we are using, we are transferring some mechanical properties of the material such as the Poisson effect to the truss topology. As such, the mechanical response is dependent on the actual connectivity. This apparent arbitrariness aims though to mimic the discrete nature of cellular tissues, which are able to change topology, and have distinct properties between the cell boundaries and the bulk material.

By including the porous-based rheological law described here to the bar elements, we aim to extend previous cell-centred models [20, 22, 23] with 
fluidisation capabilities. We adapt in the next sections the rheological model described for continua in Section 2 to the general truss system depicted in Figure 5 .

\subsection{Equilibrium equations}

The connection between two cell centres $i$ and $j$ is defined by the presence of a one-dimensional bar element wtih an associated elastic potential $U^{i j}$. The decomposition of the deformation gradient is now rewritten as a function of the current length of a bar element between cell centres $i$ and $j$, denoted by $l^{i j}=\| \boldsymbol{x}^{j}-\boldsymbol{x}^{i} \mid$, its active (or relaxed length) $L^{i j}$, and its initial length $L_{0}^{i j}=|| \boldsymbol{X}_{0}^{j}-\boldsymbol{X}_{0}^{i} \mid$. Note that the positions $\boldsymbol{x}^{a}$ after the active deformation will not be computed, and just the active length $L^{i j}$ will be included in the model as an internal variable.

In parallel with the elastic potential in Section 2.1, we define the following elastic potential of a bar joining nodes $i$ and $j$,

$$
U^{i j}=\frac{1}{2} k\left(1-c_{1} \frac{P^{i j}-P_{0}}{P_{0}}\right)\left(\varepsilon^{e, i j}\right)^{2},
$$

where $\varepsilon^{e, i j}$ is the elastic strain between cells $i$ and $j$ along direction

$$
\boldsymbol{e}^{i j}=\left(\boldsymbol{x}^{j}-\boldsymbol{x}^{j}\right) / l^{i j},
$$

and $k$ represents the stiffness of the filament. The factor $c_{1} \frac{P^{i j}-P_{0}}{P_{0}}$ represents the damage-like variable, which modulates the elastic energy through the elemental porosity variable $P^{i j}$. In our implementation we will use the following strain measure,

$$
\varepsilon^{e, i j}=\frac{l^{i j}-L^{i j}}{L^{i j}} .
$$

The elastic force along the direction $\boldsymbol{x}^{i}-\boldsymbol{x}^{j}$, denoted by $\mathbf{g}^{i j}$, is computed as $\left.\boldsymbol{e}^{i j} \frac{\partial U^{i j}}{\partial \varepsilon^{e, i j}}\right|_{L}$, which yields

$$
\mathbf{g}^{i j}=k\left(1-c_{1} \frac{P^{i j}-P_{0}}{P_{0}}\right) \varepsilon^{e, i j} \boldsymbol{e}^{i j} .
$$

The global equilibrium of the system is achieved by equalising the resultant of all the elastic forces at each cell-centre to zero, that is,

$$
\sum_{j} \mathbf{g}^{i j}=\mathbf{0}, \quad i=1, \ldots, N .
$$


These equations, and the Cauchy equilibrium equations in (3), are both equivalent to minimising the total elastic energy of the network and continua, respectively. We emphasise that the modulations of the cell elasticity with the porosity variable in (11) aims to mimic the measured relation between density of F-actin filaments and cell stiffness [19].

\subsection{Active length evolution}

The evolution law of the active strain in (4) is here rewritten in terms of the active length variable $L$ as:

$$
\dot{L}^{i j}=P^{i j} \gamma_{0} \varepsilon^{e} L^{i j}=P^{i j} \gamma_{0}\left(l^{i j}-L^{i j}\right) .
$$

The tensor like character of the active deformations is thus replaced by a scalar lengthening rate $\dot{L}^{i j}$, which is regulated by a scalar remodelling rate, $\gamma^{i j}=P^{i j} \gamma_{0}$, not necessary the same for each element $i j$. This elemental internal remodelling rate of the cytoskeleton has the same meaning as its tensor counterpart, but along the direction of the cell-cell contact. We will consider the value of $\gamma_{0}$ as constant and homogeneous throughout the cellular system. It has been demonstrated in [22] that, for a constant porosity value $P$, the evolution law in (13) can mimic the response of the Maxwell rheological model. As such, this model provides a strain and strain-dependent rate response, although in our experiments we have used a constant applied strain rate.

\subsection{Porosity evolution}

The evolution for the porosity takes in the bar system the following form:

$$
\dot{P}^{i j}=c_{2}^{ \pm}\left(P_{0}-\frac{P^{i j}}{\left(L^{i j} / L_{0}^{i j}\right)^{c 3}}\right),
$$

which is equivalent to the evolution in equation in (6) but replacing $J^{a}$ by $L^{i j} / L_{0}^{i j}$.

Like equation (6), the relation above assumesf that the rate of $P$ depends on whether $P$ increases or decreases, and on the current active deformation. Equation (14) also assumes a reference value $P_{0}$, which is employed here because the experiments simulated have a reference asymptotic configuration. According to the numerical tests shown in the next section, this value $P_{0}$ may be related to the elastic strain without altering much our results. However, 
without further experiments (sustained stretched or large cycling stretches), it is not possible to deduce a clear dependence of the reference porosity $P_{0}$ on the strains or resting length.

\subsection{Summary}

The particle based model is described by gathering the three equations in (12), (13) and (14), which may be jointly written as:

$$
\boldsymbol{f}\left(\boldsymbol{x}, L^{a}, P\right)=\mathbf{0}
$$

with

$$
\boldsymbol{f}\left(\boldsymbol{x}, L^{a}, P\right)=\left\{\begin{array}{ll}
\sum_{j} k \frac{1}{L^{i j}}\left(1-c_{1} \frac{P^{i j}-P_{0}}{P_{0}}\right) \varepsilon^{e, i j} \boldsymbol{e}^{i j}, & i=1, \ldots, N \\
\dot{L}^{i j}-\gamma_{0} P^{i j} \varepsilon^{e, i j}, & i j=1, \ldots, N_{e} \\
\dot{P}^{i j}-c_{2}^{ \pm}\left(P_{0}-\frac{P^{i j}}{\left(L^{i j} / L_{0}^{i j}\right)^{c 3}}\right), & i j=1, \ldots, N_{e}
\end{array}\right\} .
$$

After replacing the continuum model by a particle based model, we have turned the system of PDEs in (7) into the system of ODEs given above. This system is discretised on time in Appendix A using a weighting parameter $\theta$ [36]. In $n_{s d}$ space dimensions, the resulting non-linear system of equations that allows us to find the new set of $n_{s d} \times N+2 \times N_{e}$ unknowns: $\boldsymbol{x}_{n+1}^{i}, L_{n+1}^{i j}$ and $P_{n+1}^{i j}$, is solved using a Newton-Raphson scheme.

Due to the elemental character of the active lengths and porosities, these variables can be statically condensed, giving rise to a non-linear system of solely $n_{s d} \times N$ unknowns, $\boldsymbol{x}^{1}, \ldots, \boldsymbol{x}^{N}$. Appendix B explains the static condensation procedure and the system of linear equations to be solved at each Newton-Raphson iteration.

\section{Results}

\subsection{Experimental set-up}

We will here aim to reproduce the experiment carried out by X Trepat et al. [34]. In these experiments, the adherent human airway muscle (HASM) cells were cultured on a flexible substrate which is subjected to a biaxial and uniform single transient stretch during 4 seconds (see Figure 6). Before and after the stretching, the evolution of the storage and loss modulus $G^{\prime}$ and $G^{\prime \prime}$ was measured intermittently using optical magnetic twisting cytometry 
(OMTC), where magnetic microbeads attached to cytoskeleton were periodically subjected to small magnetic oscillations with frequency $2 \pi \omega=0.75 \mathrm{~Hz}$ during the subsequent 1000 seconds.

We remind that the storage and loss modulus, $G^{\prime}$ and $G^{\prime \prime}$, measure the solid-like and fluid-like response of a material, and correspond to the real and imaginary components of a complex modulus $G^{*}=G^{\prime}+i G^{\prime \prime}$ in a general rheological law $\sigma^{*}(t)=G^{*} \varepsilon^{*}(t)$ [8]. When solid elastic materials are subjected to oscillatory boundary conditions, strains $\varepsilon(t)^{*}$ and stresses $\sigma(t)^{*}$ are in phase, that is $\sigma(t)^{*}=G^{\prime} \varepsilon(t)^{*}, G^{\prime \prime}=0$ and $\delta=\operatorname{atan}\left(G^{\prime \prime} / G^{\prime}\right)=0$. Instead, stresses in fluids depend on the strain rates, so that $\sigma^{*}$ and $\varepsilon^{*}$ are delayed by a phase angle $\delta=\pi / 2$, which in the complex plane is equivalent to relate them as $\sigma(t)^{*}=i G^{\prime \prime} \varepsilon(t)^{*}$, with $G^{\prime}=0$.

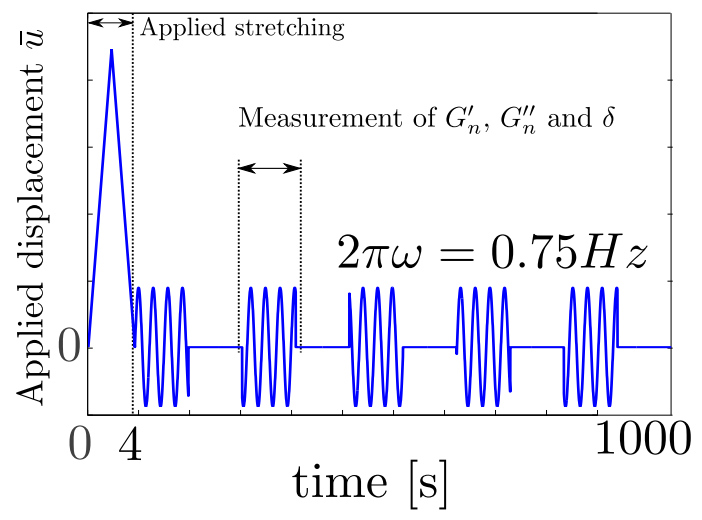

Figure 6: Schematic description of the stretching process. Cells were subjected to a single transient stretch of 4 seconds and then applying an smaller oscillatory strain of 1000 seconds with frequency $\omega=0.75 \mathrm{~Hz}$. The applied displacement $\bar{u}$ is such that the tissue is subjected to a maximum prescribed value of strain $\bar{\varepsilon}$.

In the reported experiments by Trepat et al. [34], tissue fluidisation after cessation of a single transient stretch is observed through a prompt decrease of the storage modulus $G^{\prime}$, and an increase of the phase angle $\delta$ and loss modulus $G^{\prime \prime}$, with a slow eventual recovery. In the experiments and in our simulations we have measured the normalised moduli $G_{n}^{\prime}$ and $G_{n}^{\prime \prime}$ relative to their values before the stretching, $G_{0}^{\prime}$ and $G_{0}^{\prime \prime}$. Figure 7 illustrates the experimental data of the phase angle $\delta$ (Figure 7a) and the normalised modulus $G_{n}^{\prime}=G^{\prime} / G_{0}^{\prime}$ (Figure $7 \mathrm{~b}$ ) when imposing a strain $\bar{\varepsilon}=5 \%$ (red circles) and $\bar{\varepsilon}=10 \%$ (green circles). 


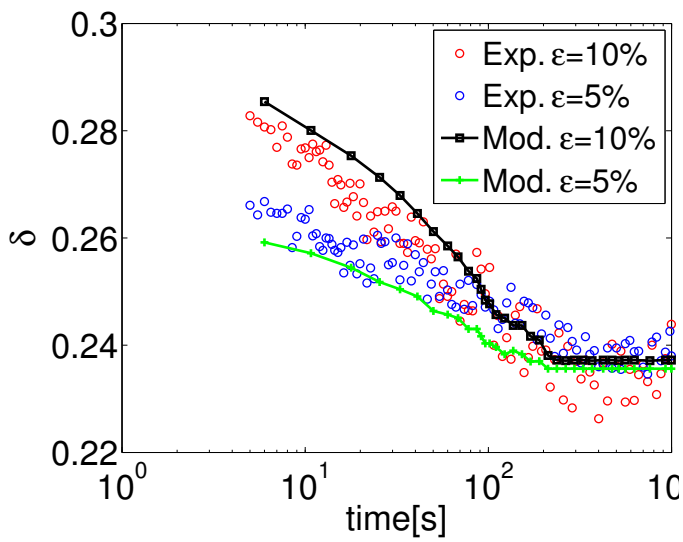

(a)

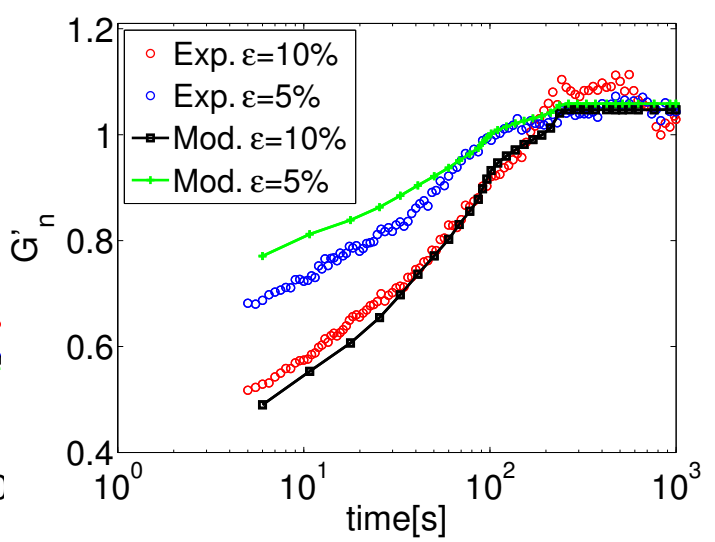

(b)

Figure 7: Evolution of the phase angle $\delta$ (a) and stored modulus $G^{\prime}$ (b) after stretch application on a single element of $\varepsilon=10 \%$ and $\varepsilon=5 \%$. Circles depict the experimental data, while continuous lines represent the results of the numerical simulation. Data is plotted from time $t=5 \mathrm{~s}$, with $t=4 \mathrm{~s}$ the end of the large stretching cycle.

\subsection{Model fitting}

After cessation the transient stretch, the phase angle $\delta$ and the normalised stiffness $G^{\prime}$ are computed in the model as follows,

$$
\delta=\tan ^{-1}\left(G_{n}^{\prime \prime} / G_{n}^{\prime}\right) \quad G_{n}^{\prime}=\frac{\sigma_{0}}{\varepsilon_{0}} \cos (\delta)
$$

with $G_{n}^{\prime}$ and $G_{n}^{\prime \prime}$ the storage and loss modulus, respectively, and with $\sigma_{0}$ and $\varepsilon_{0}$ the maximum values of the stress and strain signals. In the numerical and experimental simulations we used the oscillatory strain $\varepsilon_{0}=1 \%$. The phase angle $\delta$ increased after the single transient stretch and then slowly recovered. Instead, stored modulus $G_{n}^{\prime}$ decreased after the single transient stretch and then slowly recovered after 20 minutes, approximately. In our model, the damage-like variable $D$ in equation (8), which depends on the porosity $P$, is responsible for modulating this fluidisation effect.

The parameters of the model $p=\left\{k, \gamma_{0}, c_{1}, c_{2}^{ \pm}, c_{3}\right\}$ have been fitted in order to replicate the experimental results. The fitting has been aciheved using a least square procedure adapted from the Matlab function lsqcurvefit, 
which minimises the following functional,

$$
\begin{aligned}
f\left(k, \gamma_{0}, c_{1}, c_{2}^{ \pm}, c_{3}\right) & =\sum_{i=1}^{N}\left(\delta\left(t_{i}\right)_{10}^{E}-\delta\left(t_{i}\right)_{10}^{S}\right)^{2}+\sum_{i=1}^{N}\left(G_{n}^{\prime}\left(t_{i}\right)_{10}^{E}-G_{n}^{\prime}\left(t_{i}\right)_{10}^{S}\right)^{2} \\
& +\sum_{i=1}^{N}\left(\delta\left(t_{i}\right)_{5}^{E}-\delta\left(t_{i}\right)_{5}^{S}\right)^{2}+\sum_{i=1}^{N}\left(G_{n}^{\prime}\left(t_{i}\right)_{5}^{E}-G_{n}^{\prime}\left(t_{i}\right)_{5}^{S}\right)^{2}
\end{aligned}
$$

The superscripts $E$ and $S$ denote respectively experimental and simulated values, and the subscripts 5 and 10 correspond to values at $\bar{\varepsilon}=5 \%$ and $\bar{\varepsilon}=10 \%$. For each maximum strain, we used $N=34$ points, which required between 20 and 50 iterations in order to minimse $f\left(k, \gamma_{0}, c_{1}, c_{2}^{ \pm}, c_{3}\right)$. The model has been fitted independently for one element and multicelular systems.

\subsection{One element simulations}

In order to validate our model described in Section 2.4, we have simulated the fluidisation process by first applying the stretch history given in Figure 6 to a single bar element. We have used the fitting described in the previous section, which gave the optimal values $p^{*}=\left\{k, \gamma_{0}, c_{1}, c_{2}^{+}, c_{2}^{-}, c_{3}\right\}=$ $\{1.13,1.119,2.02,0.012,0.0012,4.75\}$. In our numerical model, we applied a displacement $\overline{\boldsymbol{u}}$ to the right side of the unit length element, while the left side remained fixed. Measurement of the moduli $G_{n}^{\prime}$ and $G_{n}^{\prime \prime}$ was achieved by applying a smaller stretch of $1 \%$ with the same experimental frequency of $75 \mathrm{~Hz}$. This strain are large enough to trigger an oscillatory stress $\sigma$, and thus measure the delay between $\varepsilon$ and $\sigma$, but are small enough to avoid altering the recovering of the $G_{n}^{\prime}$.

As shown in Figure 7, the model predictions follow the same trends of the experimental data for the fitted values. The plots in Figure 8 show the evolutions of the resting length and the porosity. While the trend of the former follows the evolution of the imposed displacement, the latter has a much slower decreasing curve. Indeed, according to equation (13), the resting length adapts to the imposed strain, while in view of equation (14), the porosity $P$ tends to decrease and equalise the reference value $P_{0}$ when $L^{a}=L_{0}$. However, due to the smaller value of $c_{2}^{-}$, this reduction of $P$ takes place at much lower rate than the increase of $P$ during the approximately first 2 seconds of the stretching cycle, where $c_{2}^{+}$is used and $\dot{P}>0$.

The different response of $L$ and $P$ is a basic ingredient of the model. While resting length $L$ follows the evolution of the applied displacement, 


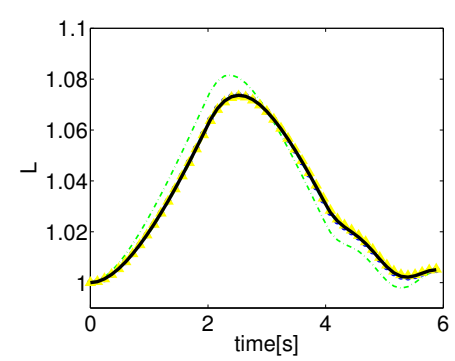

(a)

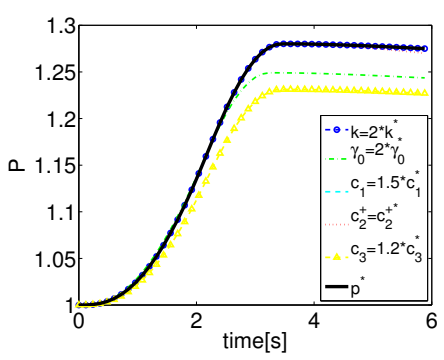

(b)

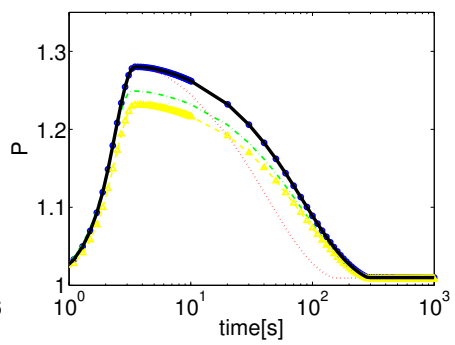

(c)

Figure 8: Time evolution of $L$ and $P$. The larger stretching cycle is applied during the first 4 seconds. (a) Evolution of resting length $L$. (b)-(c) Evolution of porosity $P$ in unscaled time units (b) and logarithmic units (c) after stretch application on a single element of $\bar{\varepsilon}=10 \%$.

due to the evolution law in (13), porosity $P$ has a much slower recovery due to the asymmetry of its evolution law in (14), which uses $c_{2}^{+}$if $\dot{P}>0$ or $c_{2}^{-}$when $\dot{P}<0$. In this sense, the resting length simulates short term visoelsticity of the polymeric structure of filaments, while $P$ represents much slower cytoskeletal reorganisation and cytosol flow.
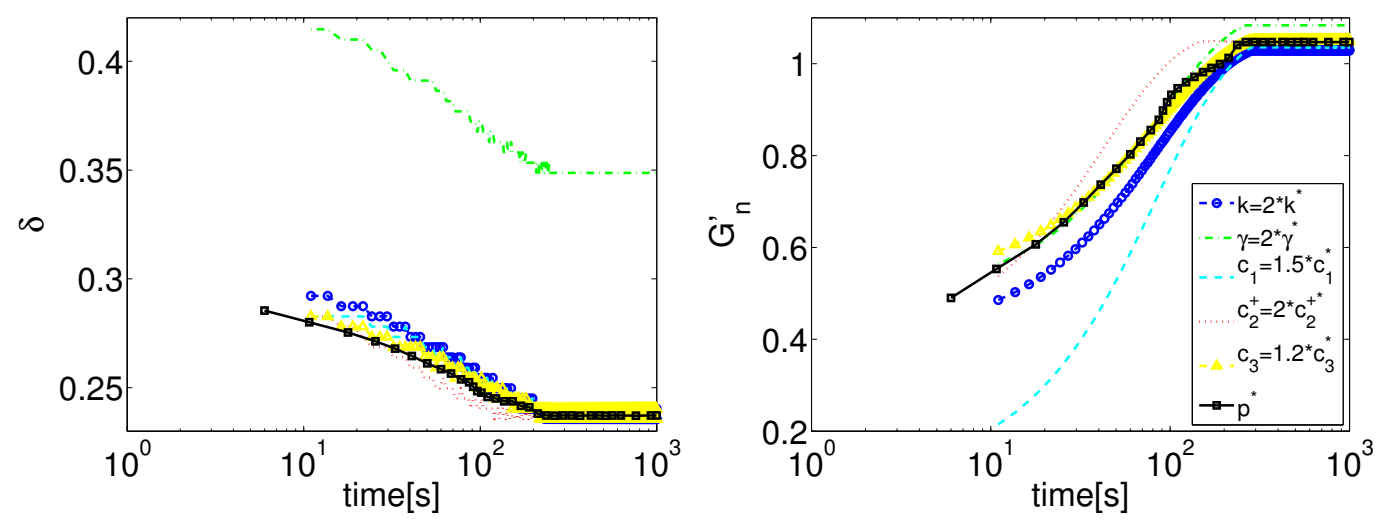

Figure 9: Evolution of $\delta$ (left) and $G_{n}^{\prime}$ (right) for different values of the fitting parameters $p=\left\{k, \gamma_{0}, c_{1}, c_{2}^{ \pm}, c_{3}\right\}$. Line indicated with $p^{*}$ correspond to optimal values, while other lines have the same values, except the parameter indicated, which is augmented according to the factor given in the legend.

Figures 8 and 9 also show the evolution of $P, L, \delta$ and $G_{n}^{\prime}$ for different parameters around the optimal values $p^{*}$. At this point, the functional showed far less sensibility with respect to the constants $k$ and $c_{3}$ than to 
the other parameters $\gamma_{0}, c_{1}$ and $c_{2}^{ \pm}$. The remodelling rate $\gamma_{0}$ determines the initial and asymptotic phase angle $\delta_{0}$, while $c_{1}$ and $c_{2}$ control the length of the recovering time and the drop of $G_{n}^{\prime}$, respectively. The stiffness value $k$ has nearly no effect, since it affects the tissue stiffness, which is normalised in our plots. Parameter $c_{3}$ also hardly affects the recovery, since it controls the dependence of $\dot{P}$ on $L$ (see equation (14)). Since the latter is fully recovered after the stretch cycle of 4 seconds, when $L \approx L_{0}$, we have that $L / L_{0} \approx 1$ and the parameter $c_{3}$ becomes irrelevant.

\subsection{Multicellular system simulations}

With the aim of extending the analysis to multicellular systems, we applied the same stretch history to the cell-centred network shown in Figure 10, where the left side was kept fully fixed, and the right side followed the displacement history in Figure 6. Figure 10a shows the deformed shape at $t=2 \mathrm{~s}$, wher the stretching is at its maximum, while Figure 10b shows the positions of the nuclei during recovery, which is very similar to the initial positions. This initial cell-centred network was obtained by pre-stressing a regular mesh of cells. This pre-stress was imposed in order to retrieve a realistic distribution of cells, which in general form hexagonal cell shapes.

The phase angle $\delta$ and the stiffness $G_{n}^{\prime}$ after cessation of the single transient stretch were computed in the same manner as previously described for the single element. $G_{n}^{\prime}$ was defined as the monolayer stiffness after stretch relative to the initial stiffness $G_{0}^{\prime}$. It can be observed in Figure 11 that using a similar set of parameters, in this case $p=\left\{k, \gamma_{0}, c_{1}, c_{2}^{+}, c_{2}^{-}, c_{3}\right\}=$ $\{0.97,1.17,2.13,0.012,0.0012,4.00\}$, the two curves corresponding to $5 \%$ and $10 \%$ stretch can be recovered. These values where obtained using the same fitting process and functional in (18) than in the one element analysis.

The trends of the porosity and resting length were also similar, but as it can be seen in Figure 10, remarkably so on the horizontal elements of the network, which are those most affected by the stretching. The same fluidistion process has been tested in other more regular networks or cell topologies formed by pentagonal shapes, with very similar trends and porosity distributions.

\section{Discussion and Conclusions}

A rheological model that exploits the changes in cell porosity has been presented. Besides mechanical equilibrium, an evolution law of the active 


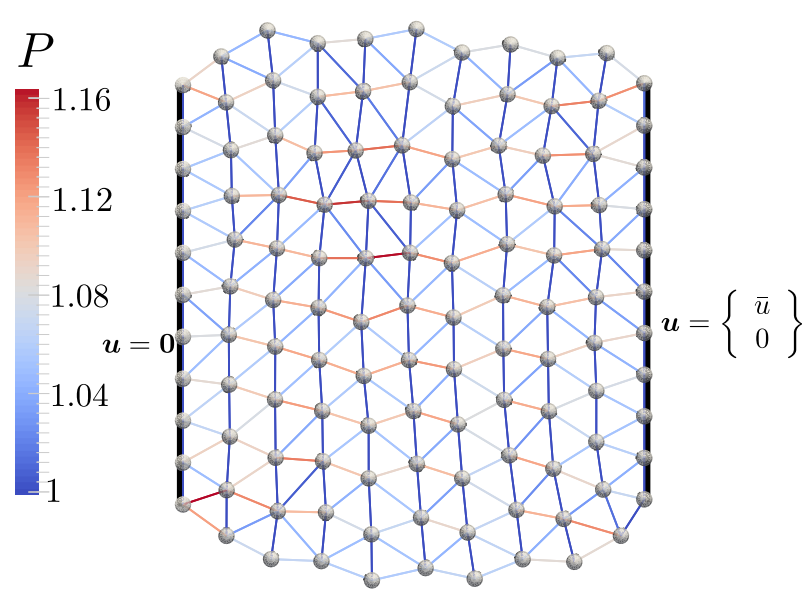

(a)

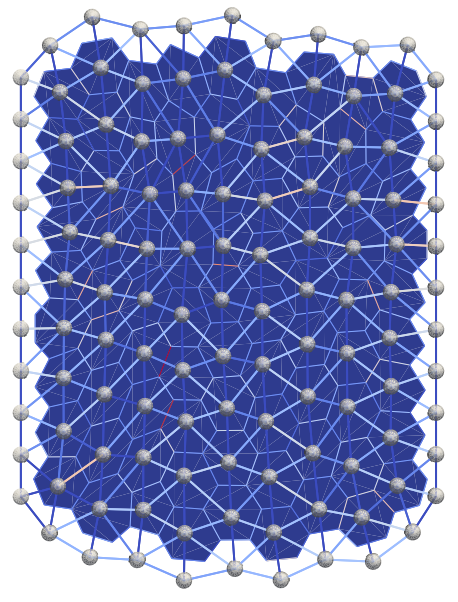

(b)

Figure 10: (a) Deformed configuration of multicellular network at time $t=2 s$ with applied boundary conditions. Bar colours indicate values of porosity $P$. (b) Configuration at time $t=150 \mathrm{~s}$ during recovery time. Cell boundaries are indicated in blue at the background.

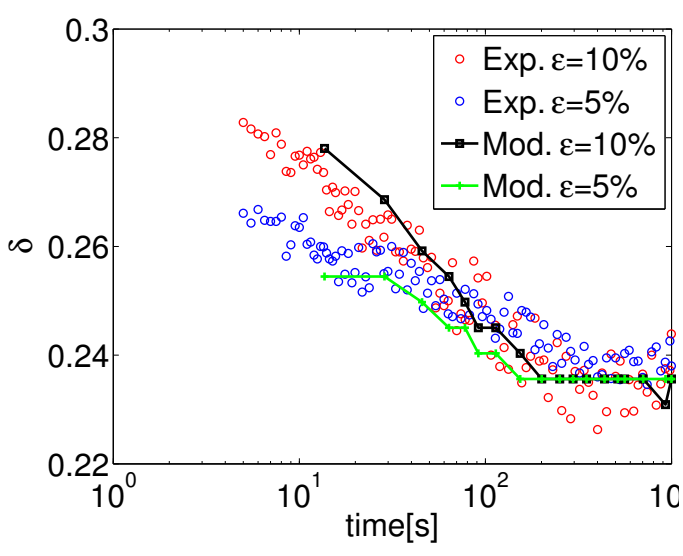

(a)

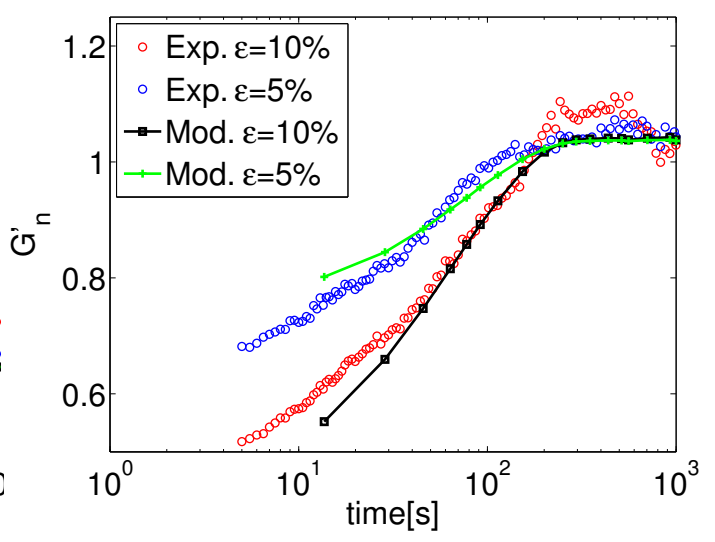

(b)

Figure 11: The response of the multicellular system to a transient stretch of $\varepsilon=10 \%$ and $\varepsilon=5 \%$. Circles depict the experimental data, and circles represent our simulation of $10 \%$ and $5 \%$ of the strain, respectively.(a) Evolution of phase angle $\delta$. (b) Evolution of stored modulus $G^{\prime}$.

configuration is used, which mimics the observed cell viscosity. We have applied the methodology to model the reversible softening and increase of the phase angle, the so-called fluidisation process observed in soft tissues. In 
the presented model, the porosity variable modulates the remodelling of the cytoskeleton, which is able to dynamically change its resting length, and also the effective mechanical response in a asimilar manner to damage models.

One of the hypotehesis behind the model is the fact that networks with larger resting length will increase their porosity, since longer filaments are increasingly distant. This assumption is not experimentally demonstrated here, and further observations are needed in order to confirm this picture. We do point out though that the correlation between the cell porosity and cell stiffness has been measured in epithelial cells [19] and in polymeric hydrogels [40]. Furthermore, longer entanglement lengths have been associated with lower stiffness. The complex interaction of cytoskeletal components and adhesive proteins renders the cell with a wide range of mechanical responses. We have not included all the possible interactions, but we represented in a coarse manner those that seem to have a prominent role, and couple them with as yet simple relationships.

The method has been applied to a single cell and multicellular systems, but can be implemented in other more complex configurations, or even tissues with changing topologies, as shown in [20]. The delayed recovery of the original mechanical properties of the tissue is due to the unsymmetrical evolution of the porosity, which is induced by the ratio $c_{2}^{+} / c_{2}^{-}$. In our simulations, this ratio was equal to 10, which implies a much slower de-polymerisation than polymerisation, cross-links formation and polymer reorganisation, i.e. porosity increases much faster than it decreases. This unsymmetrical response, which is already apparent in the experimental data recast here, needs still to be experimentally correlated with quantified (de-)polymerisation rates.

Figure 12 illustrates the physical interpretation of the different response of $L$ and $P$ during and after the stretching cycle. This two variables control the cell stiffness and the phase angle. During the stretch cyclte, resting length and porosity both increase. When compressing back the tissue, the resting length of the polymer structure is recovered, but porosity, characterised by the cross-linking of filaments and cytoskeletal reorganisation decreases at much slower pace, as indicated in Figures 12c and 12d. As porosity slowly returns to the baseline value, the tissue stiffness and solid-like response is also slowly recovered.

The evolution of porosity has been here described through a suitable law, but without explicitly modelling the filaments and their interaction. From the results reported here, more detailed models can be envisaged which may include the interaction of the cellular fluid and solid content. Furthermore, we 


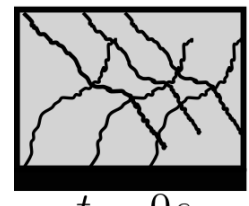

$t=0 s$

(a)

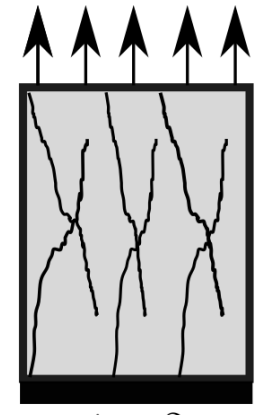

$t=2 s$

(b)

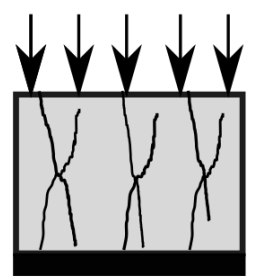

$t=4 s$

(c)

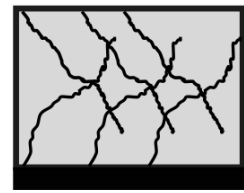

$t>>5 s$

(d)

Figure 12: Schematic of the evolution of resting length $L$ and porosity $P$. (a) Initial configuration. (b) After stretching. Increase of $L$ and $P$. (c) Just after stretching cycle. Reduction of $L$, but with no reduction in $P$. (d) During recovering. Resting length as in (b), but with reduced porosity.

have used simple linear elastic laws, which have been sufficient for simulating the simulation process. Other more sophisticated laws can be employed for including the observed softening $[3,37]$ or stiffening process $[7,9]$.

\section{Acknowledgements}

JM and NA acknowledge the support of the Ministry of Economy and Competitiveness through grant No. DPI2013-43727R, and the Generalitat de Catalunya through grant number 2014-SGR-1471. NA also acknowledges the support of Universitat Politècnica de Catalunya (UPC) and Consorci Escola Industrial de Barcelona (CEIB) through grant UPC-FPI 2012, and the European Research Council under the European Community's 7th Framework Programme (FP7/2007-2013)/ERC Grant Agreement No. 240487. XT acknowledges the support of the European Research Council under the European Union's Seventh Framework Programme (FP7/2007-2013) / ERC grant agreement 616480, the Spanish Ministry of Economy and Competitiveness (BFU2012-38146), and the Generalitat de Catalunya (2014-SGR-927). 


\section{Appendix A. Time discretisation}

The time discretisation of the evolution laws in (13) and (14) using wighted time discretisation with a paramteter $\theta[36]$ :

$$
\begin{aligned}
& L_{n+1}^{i j}=L_{n}^{i j}+\Delta t \gamma_{0} P_{n+\theta}^{i j}\left(l_{n+\theta}^{i j}-L_{n+\theta}^{i j}\right), \\
& P_{n+1}^{i j}=P_{n}^{i j}+\Delta t c_{2}^{ \pm}\left(P_{0}-\frac{P_{n+\theta}^{i j}}{\left(L_{n+\theta}^{i j} / L_{0}^{i j}\right)^{c_{3}}}\right)
\end{aligned}
$$

with $(\bullet)_{n+\theta}=(1-\theta)(\bullet)_{n}+\theta(\bullet)_{n+1}$. At each time-step $t_{n+1}$, the set of unknowns $\boldsymbol{x}_{n+1}=\left\{\boldsymbol{x}_{n+1}^{1}, \ldots, \boldsymbol{x}_{n+1}^{N}\right\}, P_{n+1}=\left\{P_{n+1}^{1}, \ldots, P_{n+1}^{N_{e}}\right\}$ and $L_{n+1}=$ $\left\{L_{n+1}^{1}, \ldots, L^{N_{e}}\right\}$ may be found by solving the following system of equations,

$$
\boldsymbol{f}\left(\boldsymbol{x}_{n+1}, L_{n+1}, P_{n+1}\right)=\mathbf{0}
$$

with

$$
\begin{aligned}
& \boldsymbol{f}\left(\boldsymbol{x}_{n+1}, L_{n+1}, P_{n+1}\right)= \\
& \quad\left\{\begin{array}{ll}
\sum_{j} k \frac{1}{L_{n+1}^{i j}}\left(1-c_{1} \frac{P_{n+1}^{i j}-P_{0}}{P_{0}}\right) \varepsilon_{n+1}^{e, i j} e_{n+1}^{i j}, & i=1, \ldots, N \\
L_{n+1}^{i j}-L_{n}^{i j}-\Delta t \gamma P_{n+\theta}^{i j}\left(l_{n+\theta}^{i j}-L_{n+\theta}^{i j}\right), & i j=1, \ldots, N_{e} \\
P_{n+1}^{i j}-P_{n}^{i j}-\Delta t c_{2}^{ \pm}\left(P_{0}-\frac{P_{n+\theta}^{i j}}{\left(L_{n+\theta}^{i j} / L_{0}\right)^{c_{3}}}\right), & i j=1, \ldots, N_{e}
\end{array}\right\}
\end{aligned}
$$

\section{Appendix B. Static condensation}

The solution of the system of equations in (A.1) may be found by using a full Newton-Raphson method. In this case, at each iteration $k$, the system of linear equations read,

$$
\mathbf{K}^{k}\left\{\begin{array}{l}
\delta \boldsymbol{x} \\
\delta L \\
\delta P
\end{array}\right\}=-\left\{\begin{array}{l}
\boldsymbol{f}_{x} \\
\boldsymbol{f}_{L} \\
\boldsymbol{f}_{P}
\end{array}\right\}_{n+1}^{k}
$$

The vectors $\boldsymbol{f}_{x}, \boldsymbol{f}_{L}$ and $\boldsymbol{f}_{P}$ denote the block of equations in vector $\boldsymbol{f}$ related to the mechanical equilibrium and the evolutions laws for $L$ and $P$. The Jacobian matrix is decomposed as follows,

$$
\mathbf{K}=\left[\begin{array}{ccc}
\mathbf{K}_{x x} & \mathbf{K}_{x L} & \mathbf{K}_{x P} \\
\mathbf{K}_{L x} & \mathbf{K}_{L L} & \mathbf{K}_{L P} \\
\mathbf{K}_{P x} & \mathbf{K}_{P L} & \mathbf{K}_{P P}
\end{array}\right]=\left\{\begin{array}{ccc}
\partial_{x} \boldsymbol{f}_{x} & \partial_{L} \boldsymbol{f}_{x} & \partial_{P} \boldsymbol{f}_{x} \\
\partial_{\boldsymbol{x}} \boldsymbol{f}_{L} & \partial_{L} \boldsymbol{f}_{L} & \partial_{P} \boldsymbol{f}_{L} \\
\partial_{\boldsymbol{x}} \boldsymbol{f}_{P} & \partial_{L} \boldsymbol{f}_{P} & \partial_{P} \boldsymbol{f}_{P}
\end{array}\right\}
$$


From the second and third block of rows in equation (B.1), the variables $\delta L$ and $\delta P$ can be computed as follow,

$$
\left\{\begin{array}{l}
\delta L \\
\delta P
\end{array}\right\}=-\mathbf{J}_{L P}^{-1}\left(\boldsymbol{f}_{L P}+\left[\begin{array}{l}
\mathbf{K}_{L x} \\
\mathbf{K}_{P x}
\end{array}\right] \delta \boldsymbol{x}\right)
$$

with $\mathbf{J}_{L P}=\left[\begin{array}{ll}\mathbf{K}_{L L} & \mathbf{K}_{L P} \\ \mathbf{K}_{P L} & \mathbf{K}_{P P}\end{array}\right]$ and $\boldsymbol{f}_{L P}=\left\{\begin{array}{l}\boldsymbol{f}_{L} \\ \boldsymbol{f}_{P}\end{array}\right\}$. Replacing the expression in (B.3) back into (B.1), yields the following reduced system of equations:

$$
\hat{\mathbf{K}}_{x} \delta \boldsymbol{x}=-\hat{\boldsymbol{f}}_{x}
$$

where the vector $\hat{\boldsymbol{f}}_{x}$ and the Jacobian $\hat{\mathbf{K}}_{x}$ are given by,

$$
\begin{aligned}
\hat{\boldsymbol{f}}_{x} & =\boldsymbol{f}_{x}-\left[\begin{array}{ll}
\mathbf{K}_{x L} & \mathbf{K}_{x p}
\end{array}\right] \mathbf{J}_{L P}^{-1} \boldsymbol{f}_{L P} \\
\hat{\mathbf{K}}_{x} & =\mathbf{K}_{x x}-\left[\begin{array}{ll}
\mathbf{K}_{x L} & \mathbf{K}_{x p}
\end{array}\right] \mathbf{J}_{L P}^{-1}\left[\begin{array}{l}
\mathbf{K}_{L x} \\
\mathbf{K}_{p x}
\end{array}\right] .
\end{aligned}
$$

The condensed system of equation in (B.4) can be now solved by NewtonRaphson method. Note that since variables $L$ and $P$ are elemental, matrix $\mathbf{J}_{L P}$ is formed by $N_{e}$ uncoupled matrices with dimensions $2 \times 2$, and therefore the products in (B.5) are computationally cheap.

\section{References}

[1] D. Azevedo, M. Antunes, S. Prag, X. Ma, U. Hacker, G.W. Brodland, M.S. Hutson, J. Solon, and A. Jacinto. DRhoGEF2 regulates cellular tension and cell pulsations in the amnioserosa during Drosophila dorsal closure. PLOS ONE, 6(9):e23964, 2011.

[2] B. Calvo, E. Peña, M. A. Martinez, and M. Doblaré. An uncoupled directional damage model for fibred biological soft tissues. formulation and computational aspects. Int. J. Num. Meth. Engng., 7(9):e1002153, 2011.

[3] O. Chaudhuri, S.H. Parekh, and D.A. Fletcher. Reversible stress softening of actin networks. Nature, 445:295-298, 2007.

[4] C. Chen, R. Krishnan, E. Zhou, A. Ramachandran, D. Tambe, K. Rajendran, R.M. Adam, L. Deng, and J.J. Fredberg. Fluidization and resolidification of the human bladder smooth muscle cell in response to transient stretch. PLOS ONE, 5:16-21, 2010. 
[5] B.N. Cox and D.W. Smith. On strain and stress in living cells. J. Mech. Phys. Solids., 71(14):239-252, 2014.

[6] G.E. Fantner, E. Oroudjev, L.S. Golde G. Schitter, P.Thurner, M.M. Finch, P. Turner, T. Gutsmann, D.E. Morse, H. Hansma, and P.K. Hansma. Sacrificial bonds and hidden length: unraveling molecular mesostructures in tough materials. Bioph. J., 90(4):1411-1418, 2006.

[7] P. Fernández, P.A. Pullarkat, and A. Ott. A master relation defines the nonlinear viscoelasticity of single fibroblasts. Bioph. J., 90:3796-3805, 2006.

[8] W N Findley, K Onaran, and W J Lai. Creep and Relaxation of Nonlinear Viscoelastic Materials: With an Introduction to Linear Viscoelasticity. Dover Publications, 1989.

[9] M.L. Gardel, J.H. Shin, F.C. MacKintosh, L. Mahadevan, P. Matsudaira, and D.A. Weitz. Elastic behavior of cross-linked and bundled actin networks. Science, 304:1301-1305, 2004.

[10] K. Garikipati, J.E. Olberding, H. Narayanan, E. M. Arruda, K. Grosh, and S. Calve. Biological remodelling: stationary energy, configurational change, internal variables and dissipation. J. Mech. Phys. Solids., 54:1493-1515, 2006. arXiv:q-bio/0506023v2.

[11] G.A. Holzapfel. Nonlinear solid mechanics. A continuum approach for engineers. J Wiley \& Sons Ltd, Chichester., 2000.

[12] P. Kollmannsberger and B. Fabry. Active soft glassy rheology of adherent cells. Soft Matter, 5:1771-1774, 2009.

[13] R. Krishnan, C.Y. Park, Y.C. Lin, J. Mead, R.T. Jaspers, X. Trepat, G. Lenormand, D. Tambe, A.V. Smolensky, A.H. Knoll, J.P. Butler, and J.J. Fredberg. Reinforcement versus fluidization in cytoskeletal mechanoresponsiveness. PLOS ONE, 4:e5486, 2009.

[14] O Lieleg, KM Schmoller, CJ Cyron, Y Luan, WA Wall, and AR Bausch. Structural polymorphism in heterogeneous cytoskeletal networks. Soft Matter, 5:1796-1803, 2009. 
[15] X. Ma, H.E. Lynch, P.C. Scully, and M.S. Hutson. Probing embryonic tissue mechanics with laser hole drilling. PB, 6:036004, 2009.

[16] E. Maher, A. Creane, C. Lally, and D. Kelly. An anisotropic inelastic constitutive model to describe stress softening and permanent deformation in arterial tissue. J. Mech. Behav. Biomed. Mater., 12:9-19, 2012.

[17] M. Maraldi and K. Garikipati. The mechanochemistry of cytoskeletal force generation. Biomech. Model. Mechanobiol., 14:59-72, 2015.

[18] J.E. Marsden and T.J.R. Hughes. Mathematical foundations of elasticity. Dover Publications, 1994.

[19] E. Moeendarbary, L. Valon, M. Fritzsche, A.R. Harris, D.A. Moulding, A.J. Thrasher, E. Stride, L. Mahadevan, and G.T. Charras. The cytoplasm of living cells behaves as a poroelastic material. Numer. Math., 12:253-261, 2013.

[20] P. Mosaffa, N. Asadipour, D Millán, A. Rodríguez-Ferran, and J.J. Muñoz. Cell-centred model for the simulation of curved cellular monolayers . Comp. Part. Mech., 2(4):359-370, 2015.

[21] J J Muñoz, K Barrett, and M Miodownik. A deformation gradient decomposition method for the analysis of the mechanics of morphogenesis. J. Biomechanics, pages 1372-1380, 2007.

[22] J.J. Muñoz and S. Albo. Physiology-based model of cell viscoelasticity. Phys. Rev. E, 88(1):012708, 2013.

[23] J.J. Muñoz, V. Conte, N. Asadipour, and M. Miodownik. A truss element for modelling reversible softening in living tissues. Mech. Res. Comm., 49:44-49, 2013.

[24] E. Peña. Damage functions of the internal variables for soft biological fibred tissues. Mech. Res. Comm., 38:610-615, 2011.

[25] E. Peña. Prediction of the softening and damage effects with permanent set in fibrous biological materials. J. Mech. Phys. Solids., 59(9):18081822, 2011. 
[26] H.J. Qi and M.C. Boyce. Constitutive model for stretch-induced softening of the stress-stretch behavior of elastomeric materials. J. Mech. Phys. Solids., 52(10):2187-2205, 2004.

[27] J. Ranft, M. Basan, J. Elgeti, J.F. Joanny, J. Prost, and F. Jülicher. Fluidization of tissues by cell division and apoptosis. Proc. Nat. Acad. Sci. USA, 107(49):20863-8, 2008.

[28] S Reese and S Govindjee. Theoretical and numerical aspects in the thermo-viscoelastic material behaviour of rubber-like polymers. Mech. Time-Dep. Mat., 1:357-396, 1998.

[29] E.K. Rodriguez, A. Hoger, and A.D. McCulloch. Stress-dependent finite growth in soft elastic tissues. J. Biomechanics, 27:455-467, 1994.

[30] J.F. Rodríguez, F. Cacho, J.A. Bea, and M. Doblaré. A stochasticstructurally based three dimensional finite-strain damage model for fibrous soft tissue. J. Mech. Phys. Solids., 54(9):864-886, 2006.

[31] C. Semmrich, R.J. Larsen, and A.R. Bausch. Nonlinear mechanics of entangled F-actin solutions. Soft Matter, 4:1675-1680, 2008.

[32] C. Semmrich, T. Storz, J. Glaser, R. Merkel, A.R. Bausch, and K. Kroy. Glass transition and rheological redundancy in F-actin solutions. Proc. Nat. Acad. Sci. USA, 104(51):20199-20203, 2007.

[33] C. Storm, J.J. Pastore, F.C. MacKintosh, T.C. Lubensky, and P.A. Janmey. Nonlinear elasticity in biological gels. Nature, 435:191-194, 2005.

[34] X. Trepat, L. Deng, S.S. An, D. Navajas, D.J. Tschumperlin, W.T. Gerthoffer, J.P. Butler, and J.J. Fredberg. Universal physical responses to stretch in the living cell. Nature, 447(3):592-596, 2007.

[35] X. Wang and W. Hong. A visco-poroelastic theory for polymeric gels. Proc. Royal Soc. A, 468:3824-3841, 2012.

[36] E.L. Wilson. A computer program for the dynamic stress analysis of underground structures. Technical report, Division of Structual Engineering Structural Mechanics, University of California, Berkeley, 1968. 
[37] L. Wolff, P. Fernández, and K. Kroy. Resolving the Stiffening-Softening Paradox in Cell Mechanics. PLOS ONE, 7(7):e40063, 2012.

[38] J. Xu, Y. Tseng, and D. Wirtz. Strain hardening of actin filament networks. Regulation by the dynamic cross-linking protein alpha-actinin. J. Biol. Chem., 275:35886-35892, 2000.

[39] M. Yang and L.A. Taber. The possible role of poroelasticity in the apparent viscoelastic behavior of passive cardiac muscle. J. Biomechanics, 24(7):587-597, 1991.

[40] O. Yom-Tov, L. Neufeld, D. Seliktar, and H. Bianco-Peled. A novel design of injectable porous hydrogels with in situ pore formation. Acta Biomat., 10(10):4236-4246, 2014. 